\title{
Laboratory Measurements of a Steady Breaker Using PIV and BIV
}

\author{
Chang Lin, ${ }^{1}$ Shih-Chun Hsieh, ${ }^{1}$ I-Ju Lin, ${ }^{1}$ Kuang-An Chang, ${ }^{2}$ and Rajkumar Raikar ${ }^{3}$
}

Image-based particle image velocimetry (PIV) and bubble image velocimetry (BIV) techniques were employed to measure the flow field in both the non-aerated and aerated regions in a steady hydraulic jump. The jump has a Froude number of 4.62 with a large amount of air entrainment. The mean velocities and turbulence properties were obtained by ensemble averaging the repeated measurements. The spatial variations of mean velocity profiles, turbulent intensity, and Reynolds stress were discussed in details. The results show that the ratio between the maximum bubble velocity and the maximum water velocity is almost constant with a value about 0.6 . The turbulence intensity of bubbles is very high in the aerated region and reaches about 0.4 , while the turbulence velocity of water below the roller is significantly lower. The Reynolds stress is mostly negative and increases as elevation increases. The maximum Reynolds stress occurs at the free surface.

\section{Introduction}

A hydraulic jump is a natural phenomenon that is usually seen in a river or downstream of a hydraulic structure such as a sluice gate or a spillway. A hydraulic jump occurs when a supercritical flow changes abruptly into a subcritical flow. Previous studies on hydraulic jumps can be roughly classified into two categories: the earlier external flow geometry and the more recent internal flow structure. The earlier description of hydraulic jumps mainly focused on the measurement of surface profile and depth-length relation of the jumps (Rajaratnam and Subramanya 1968, Leutheusser and Kartha 1972, Mehrotra 1976). Studies on the internal flow structure of hydraulic jumps have been focused on the velocity field, turbulent characteristics, and void fraction in the jumps. Rajaratnam (1965) conducted velocity measurements using a pitot tube in a hydraulic jump and concluded that the velocity distribution in a hydraulic jump is similar to that in a typical wall jet. Liu et al. (2004) used acoustic Doppler velocimetry (ADV) to measure velocity, turbulence intensity, and Reynolds stresses in hydraulic jumps and found that turbulence intensity and Reynolds stress have some degrees of similarity. Long et al. (1990) used LDV to measure the mean velocity, turbulent shear stresses, and turbulence intensity for a submerged hydraulic jump. Lennon and Hill (2006) used PIV to measure mean and turbulent

\footnotetext{
${ }^{1}$ Department of Civil Engineering, National Chung Hsing University, Taichung 402, Taiwan

${ }^{2}$ Zachry Department of Civil Engineering, Texas A\&M University, College Station, Texas 77843-3136, USA. E-mail: kchang@tamu.edu

${ }^{3}$ Department of Civil Engineering, K.L.E.S. College of Engineering and Technology, Belgaum 590008, India
} 
velocity fields and demonstrated the structure of jumps for Froude numbers ranging from 1.4 to 3.0 .

Studies in the aerated region of a hydraulic jump have been mostly focused on the air entrainment process and the quantity of entrained air bubbles. Chanson and Brattberg (2000) studied the air-water flow properties in the shear region of hydraulic jumps using dual-tip conductivity probes. Murzyn et al. (2005) examined the bubble characteristics using an optical probe and compared the results with the measurements by Chanson and Brattberg (2000). Chanson (2007) reviewed the recent advances in hydraulic jumps.

Recently, Ryu et al. (2005) developed a method called bubble image velocimetry (BIV) that combines the PIV technique and the shadowgraphy method for velocity measurements in the highly aerated region. Using BIV, Lin et al. (2008) successfully measured the velocity fields inside and outside the aerated region of a periodic oscillatory flow over a vertical drop pool. The objective of the present study is to investigate the flow structure in the aerated region of hydraulic jumps using BIV. In addition, the velocity field outside the aerated region will be measured using PIV.

\section{Experimental Setup}

Experiments were conducted in a recirculating water flume at the Department of Civil Engineering, National Chung Hsing University. The internal dimensions of the test section are $3.05 \mathrm{~m}$ long, $0.5 \mathrm{~m}$ wide, and $0.54 \mathrm{~m}$ deep as shown in Lin et al. (2008). Both the PIV system, including the laser light source and the high-speed camera, and the BIV system are also identical to that in Lin et al.

A sluice gate was installed in the flume to generate the desired hydraulic jump. In the present study, the hydraulic jump has an approach velocity of $U_{1}=196.3 \mathrm{~cm} / \mathrm{s}$ and conjugate water depths of $y_{1}=1.84 \mathrm{~cm}$ and $y_{2}=11.2 \mathrm{~cm}$. The Froude number, defined as $F r=U_{1} / \sqrt{g y_{1}}$ with $g$ being the gravitational acceleration, is $F r=4.62$. The hydraulic jump is a steady jump therefore the mean and turbulence properties can be obtained directly by ensemble averaging the repeated measurements.

The coordinate system is defined as follows: the origin is defined as $x=0$ being at the toe of the hydraulic jump and $y=0$ at the bottom of the tank. In the present study, images from four fields of view (FOVs) were taken using the PIV system. The FOVs cover the range from $x<0$ to $x=$ $26 \mathrm{~cm}\left(x / y_{1}=14.1\right)$. In addition, images were taken from four FOVs using the BIV system, covering the range from $x<0$ to $x=40 \mathrm{~cm}\left(x / y_{1}=21.7\right)$. The sketch of the FOVs is shown in Fig. 1. The size of the FOVs in the PIV measurements is $7.5 \mathrm{~cm} \times 3.75 \mathrm{~cm}$ while they are 17.0 $\mathrm{cm} \times 8.5 \mathrm{~cm}$ and $17.0 \mathrm{~cm} \times 12.75 \mathrm{~cm}$ in the BIV measurements. The time interval between the 
recorded images is $1.0 \mathrm{~ms}$ in both PIV and BIV measurements. The mean velocity was calculated from ensemble averaging 16,000 to 19,000 instantaneous velocity fields from repeated runs with the same test condition in both PIV and BIV measurements. Reynolds decomposition was used to separate the instantaneous velocity into mean and fluctuations, i.e., $u_{i}=\left\langle u_{i}\right\rangle+u_{i}^{\prime}$, in which $u_{i}$ is the instantaneous velocity, $\left\langle u_{i}\right\rangle$ is the mean velocity, $u_{i}^{\prime}$ is the fluctuations, the subscript $i$ is the direction, and $\langle\cdot\rangle$ is the ensemble- average operator.

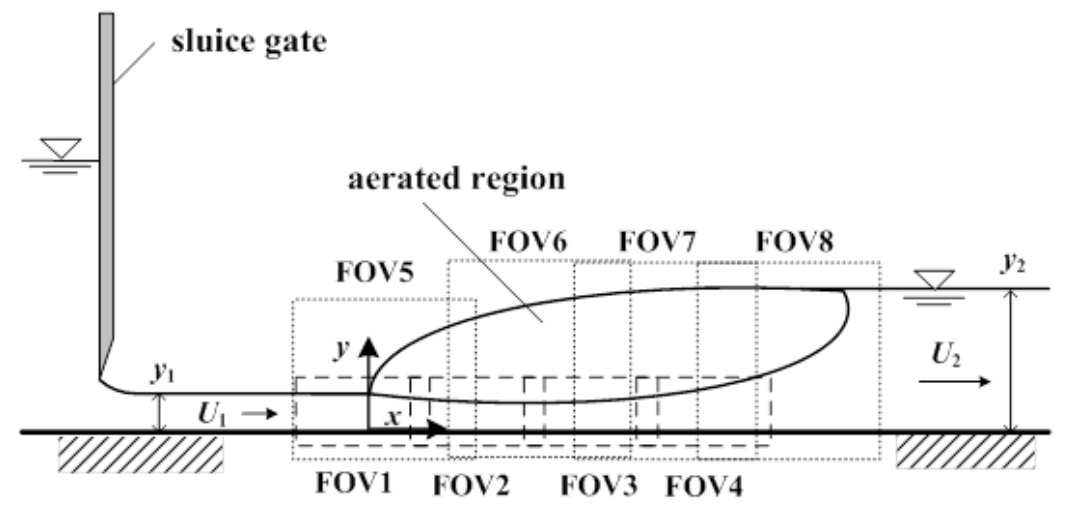

Fig. 1. Definitions of variables and coordinate system and fields of view for PIV and BIV measurements

\section{Results and Discussion}

The velocity field in the hydraulic jump was measured separately using the PIV and BIV techniques for the water region and the aerated region, respectively. The mean BIV image is shown in Fig. 2(a). It shows the area covered by bubbles in the jump as well as the free surface before the jump. The mean image consists of four FOVs so the average gray value of each FOV may not be the same. The corresponding mean velocity, normalized by the depth-averaged upstream velocity $U_{1}$, is shown in Fig. 2(b). Note that the PIV measured velocity was plotted together with the BIV measured velocity in the figure. From the figure, there is a certain region in which both the PIV and BIV techniques are able to result in velocities. This region is indeed has a lower concentration of bubbles (observed through video images) and the bubbles are somewhat intermittently being convected downward. The velocities in this overlapped region are not the same because PIV measures the water velocity while BIV measures the bubble velocity. This will be further discussed later. The mean vorticity field, normalized by $U_{1} / y_{1}$, corresponding to the BIV measured mean velocity is shown in Fig. 2(c). As expected, the vorticity level is quite high at the lower boundary of the aerated region, indicating a large counterclockwise roller in this region that is also observed from the mean velocity field. 
(a)

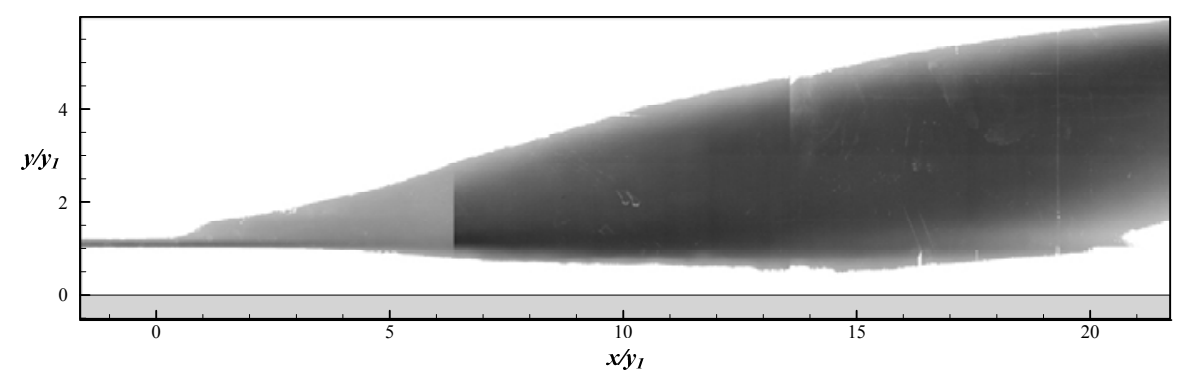

(b)

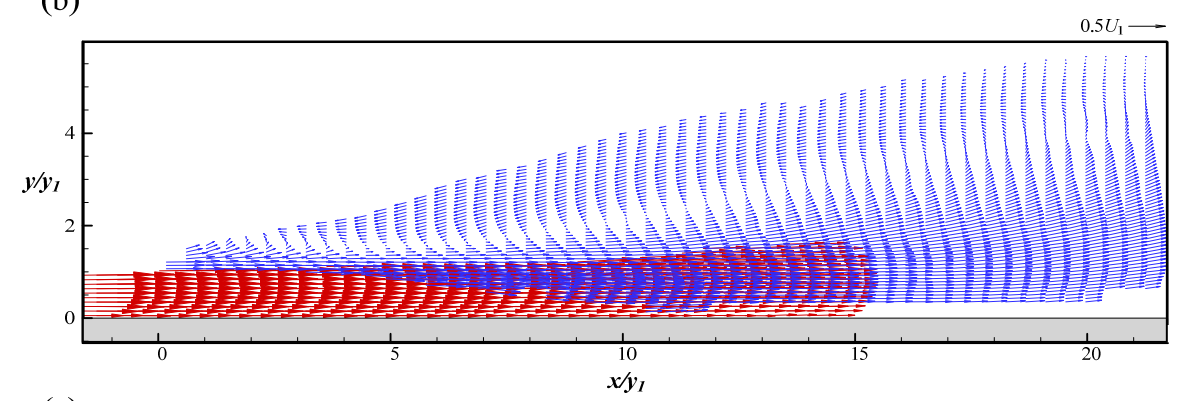

(c)

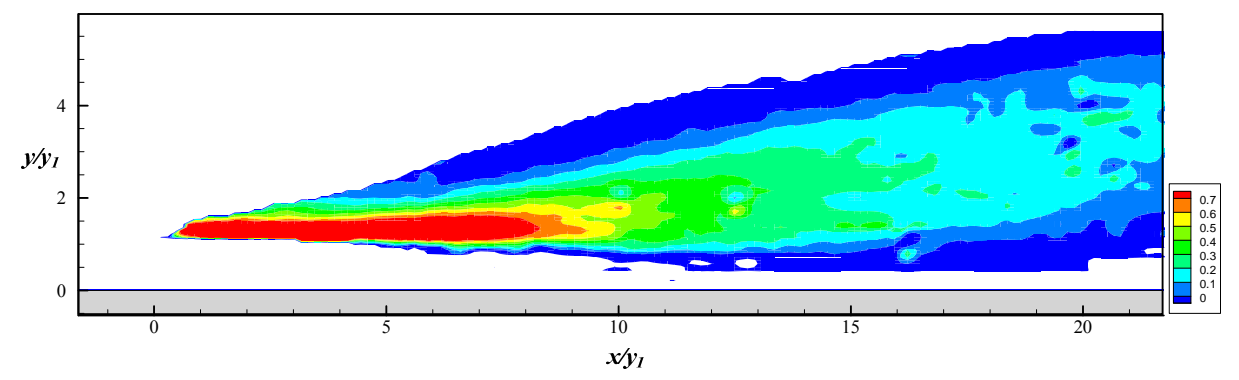

Fig. 2. (a) Mean BIV image; (b) mean velocity field $\langle\vec{u}\rangle / U_{1}$ with the PIV measured velocity (in red) plotted below the BIV measured velocity (in blue); (c) mean vorticity field $\omega /\left(U_{1} / y_{1}\right)$.

Fig. 3(a) shows the detailed mean horizontal velocity profile, $\langle u\rangle / U_{1}$, at different cross sections downstream from the toe, and Fig. 3(b) shows the corresponding mean vertical velocity profile, $\langle v\rangle / U_{1}$. Both the horizontal and vertical velocities measured by PIV and BIV were plotted. As mentioned earlier, there is an overlap region in which both the PIV and BIV techniques were able to obtain velocities due to the relative sparse bubble concentration. In Fig. 3(a), the maximum horizontal water velocity remains reaching $\langle u\rangle / U_{1}=1$ until $x / y_{1}=10$, and starts to decrease after that. In Fig. 3(b), the maximum vertical water velocity is nonzero near the surface at the toe of jump at $x / y_{1}=0$, and increases as $x / y_{1}$ increases. Based on Fig. 3, the water velocity profile shows the tendency of deflecting upward underneath the roller region from the toe of the jump, somewhat similar a plane wall jet detaching from the plane boundary. 
(a)
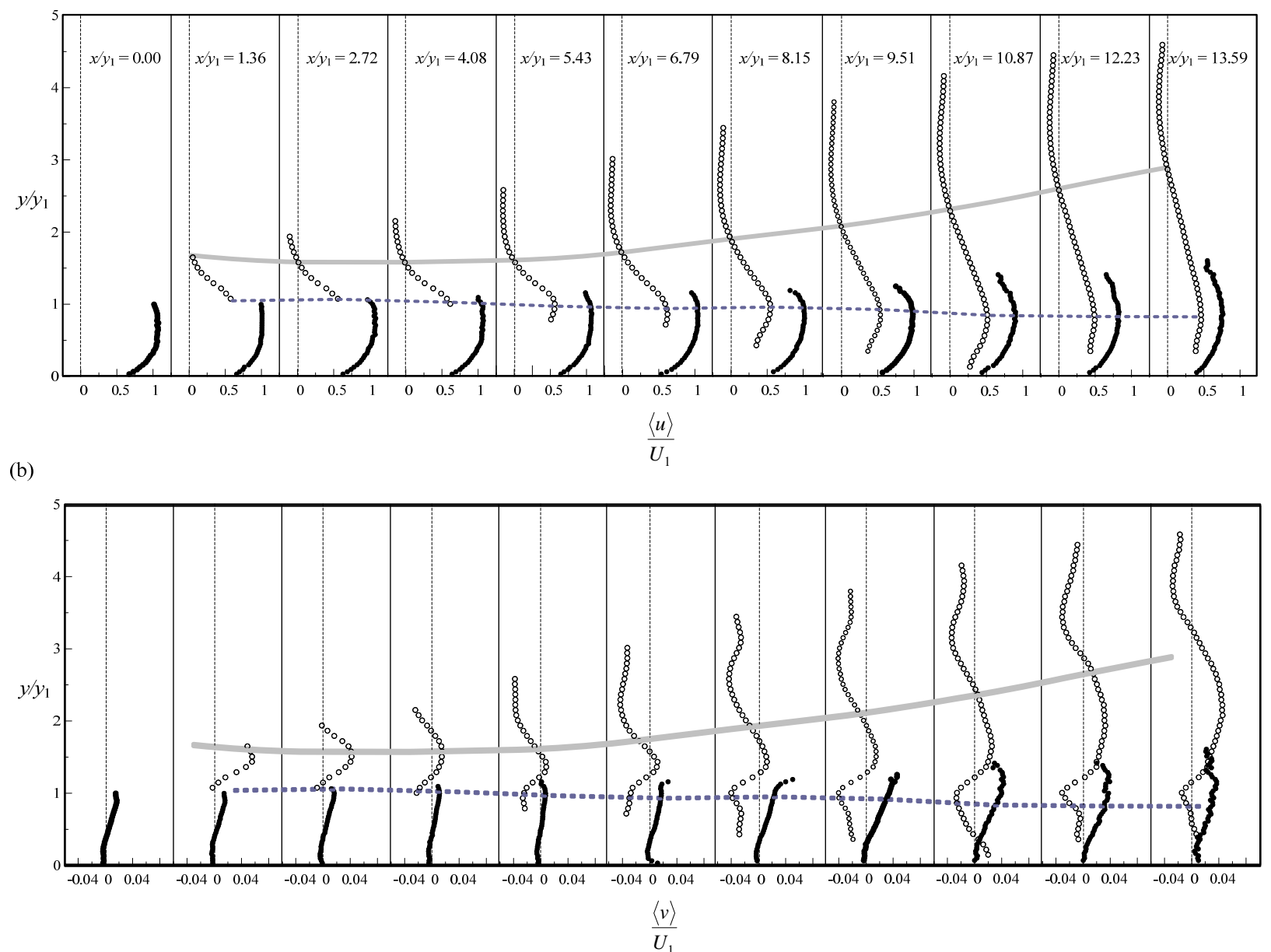

Fig. 3. Mean velocity distribution at difference downstream locations: •, water; o, bubble. (a) $\langle u\rangle / U_{1}$, (b) $\langle v\rangle / U_{1}$. The thick solid line connects the points of zero velocity while the thick dashed line connects the points of maximum velocity in the aerated region.

Fig. 4 plots the corresponding ratio of the maximum bubble velocity versus the maximum water velocity. Interestingly, the ratio is almost constant with an average value close to 0.6 . Note that this ratio may depend on the Froude number and physical dimensions of the jump therefore more investigation is needed. As shown in Fig. 4, the mean horizontal velocity of bubbles is only about 0.6 times that of water in this region. Based on our observation from the video images and observation in Chanson (2007), most bubbles are entrained at the toe of the jump and carried downstream by the flow. A certain number of bubbles are then "rolled" upstream by the reverse flow at the upper region of the roller and degassed. In the recirculation process, some bubbles are carried downward to the overlap region, intermittently, and ejected to the lower part of the jump. They therefore have a negative vertical velocity as observed in Fig. 3(b). 


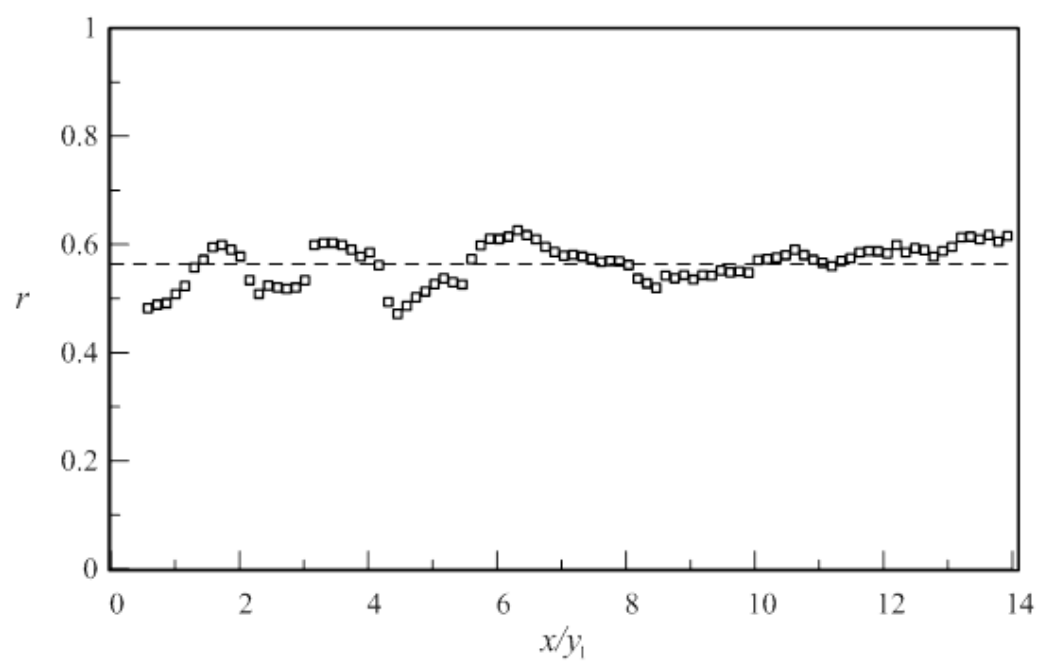

Fig. 4. Ratio of maximum bubble horizontal velocity and corresponding water velocity. The dashed line is the average that has a value about 0.6.

The turbulence properties, including velocity fluctuations in the $x$ and $y$ directions, turbulence intensity, and Reynolds stress, extracted from the bubble velocities are plotted in Fig. 5. Fig. 5(a) shows the normalized $x$-direction root-mean-square (r.m.s.) velocity fluctuation, $\sqrt{\left\langle u^{\prime 2}\right\rangle} / U_{1}$, while Fig. 5(b) shows the $y$-direction r.m.s. velocity fluctuation, $\sqrt{\left\langle v^{\prime 2}\right\rangle} / U_{1}$. In Fig. 5(a), the magnitude of $\sqrt{\left\langle u^{\prime 2}\right\rangle}$ is quite large at the lower aerated region from toe to $x / y_{1}=15$ with its maximum reaching about $0.4 U_{1}$. The region with the largest $\sqrt{\left\langle u^{\prime 2}\right\rangle}$ does not align with the region of maximum mean velocity but above the region of maximum velocity. The magnitude of $\sqrt{\left\langle v^{\prime 2}\right\rangle} / U_{1}$, shown in Fig. 5(b), is relatively smaller in comparison to that of $\sqrt{\left\langle u^{\prime 2}\right\rangle} / U_{1}$, especially near the toe. Unlike the magnitude of $\sqrt{\left\langle u^{\prime 2}\right\rangle} / U_{1}$ is quite constant along the jump, the magnitude of $\sqrt{\left\langle v^{\prime 2}\right\rangle}$ is quite small near the toe and gradually picks up and reaches the maximum at $x / y_{1} \approx 13$. The maximum value of $\sqrt{\left\langle v^{\prime 2}\right\rangle}$ is about $0.3 U_{1}$.

The turbulence intensity, defined as $\sqrt{\left\langle u^{\prime 2}+v^{\prime 2}\right\rangle} / U_{1}$, is shown in Fig. 5(c). As expected, the pattern is indeed the combination of the velocity fluctuations in the $x$ and $y$ directions. The pattern is somewhat similar to the shear layer of a plane wall jet in which the maximum turbulence level occurs in between the maximum mean velocity and the zero mean velocity (Rajaratnam 1976). Moreover, it is similar to the shear layer with its thickness and the vertical 
velocity fluctuations increasing as the horizontal distance increases. However, unlike a shear layer, the turbulence intensity is nearly constant along a vertical axis above the center of the roller, while the mean velocity is quite small but near constant as well.

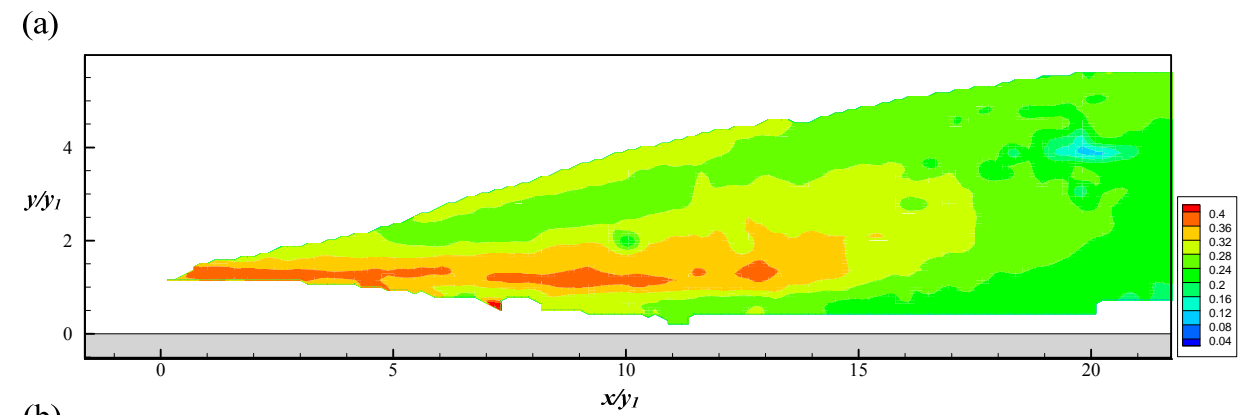

(b)

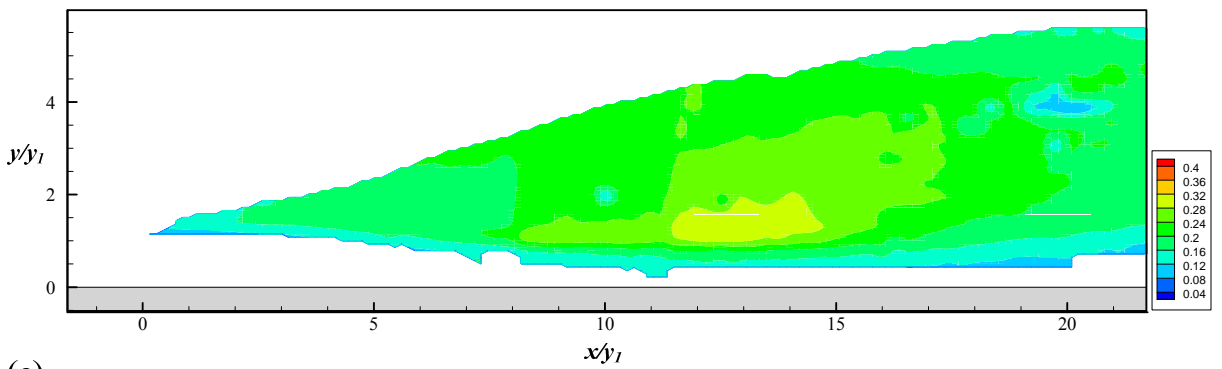

(c)

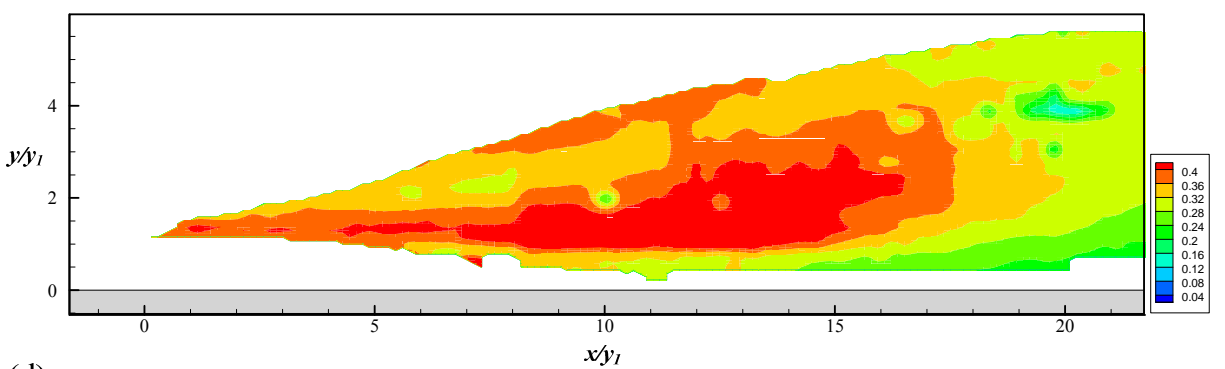

(d)

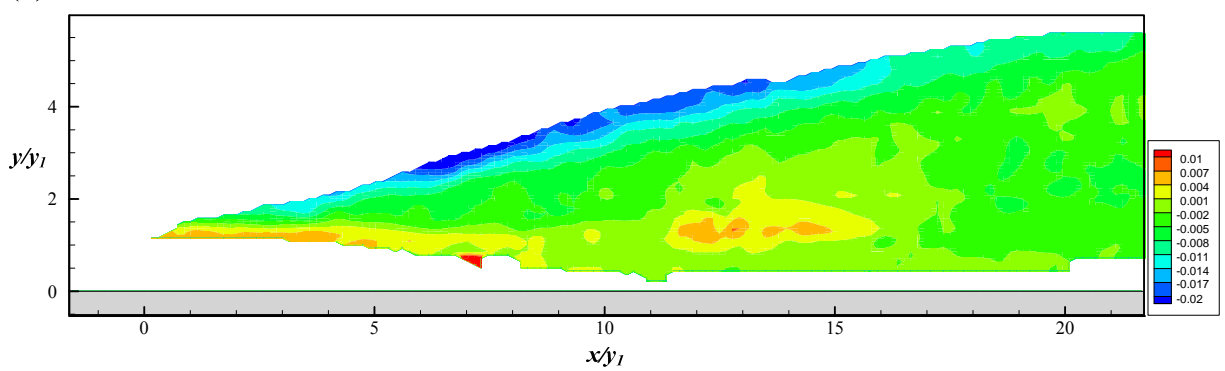

Fig. 5. (a) $x$-direction velocity fluctuation $\left\langle u^{\prime 2}\right\rangle^{1 / 2} / U_{1}$, (b) $y$-direction velocity fluctuation $\left\langle v^{\prime 2}\right\rangle^{1 / 2} / U_{1}$, (c) turbulence intensity $\left\langle u^{\prime 2}+v^{\prime 2}\right\rangle^{1 / 2} / U_{1}$, (d) Reynolds stress $-\left\langle u^{\prime} v^{\prime}\right\rangle / U_{1}^{2}$. 
The normalized Reynolds (shear) stress (without the fluid density), $-\left\langle u^{\prime} v^{\prime}\right\rangle / U_{1}^{2}$, is shown in Fig. 5(d). The stress is negative above the center of the roller with its magnitude increasing when elevation increases and, surprisingly, reaching the maximum at the free surface. Since the free surface is not expected to cause large friction therefore the stress should contribute from the internal friction between the bubbles and water. The stress changes from negative to positive near the middle between the center of the roller and the line of the maximum mean velocity. As expected, the stress below the roller in the water region is quite small. In the overlap region, the sign of stress is negative for water but positive for bubbles and the magnitude of stress between water and bubble in this region is very close to each other. This may indicate the stress is caused by each other due to their velocity difference.

\section{Conclusions}

Based on the measurements and video observation, air bubbles are entrained from the toe of the jump, and the aerated (roller) region of the jump is then formed. The roller recirculates with an inclined centerline and turbulent nature. In the overlapped region between aerated and water regions, the maximum velocities of water and bubbles are different even though the locations are coincident. The ratio between the maximum bubble velocity and the maximum water velocity is almost constant with an average value about 0.6 .

The $x$-direction velocity fluctuation, $\sqrt{\left\langle u^{\prime 2}\right\rangle}$, is very high at the lower aerated region from the toe to approximately $x / y_{1}=15$ with a maximum of about $0.4 U_{1}$. The magnitude of the $y-$ direction velocity fluctuation, $\sqrt{\left\langle v^{\prime 2}\right\rangle}$, is relatively lower at the same region, especially near the toe. There exists a big gap between the turbulent intensity, $\sqrt{\left\langle u^{\prime 2}+v^{\prime 2}\right\rangle} / U_{1}$, of water and that of bubbles, and this gap gradually decreases as $x$ increases. The gap reaches as large as $0.3 U_{1}$ near the toe. It shows that bubbles fluctuate violently when they are entrained near the toe or are recirculating in the roller, even though their mean velocity is much less than that of water, implying bubbles move slower but fluctuate a lot in the overlap region. The Reynolds stress is mostly negative, especially above the center of the roller. Its magnitude increases as the elevation increases, and reaches the maximum value at the free surface.

\section{Acknowledgments}

The authors gratefully thank the financial support by the National Science Council of Taiwan under Grant Nos. NSC 99-2221-E-005-117-MY3 and NSC 100-2119-M-005-002, and by the Directorate General of the Highways Bureau, Ministry of Transportation and Communication of Taiwan. KA Chang also wishes to thank financial support from the State Key Laboratory of Hydraulics and Mountain River Engineering, Sichuan University for attending the conference. 


\section{References}

Chanson, H., and Brattberg, T. (2000). "Experimental study of the air-water shear flow in a hydraulic jump." International Journal of Multiphase Flow, 26, 583-607.

Chanson, H. (2007). "Hydraulic Jumps: Bubbles and Bores." Proceedings of the $16^{\text {th }}$ Australasian Fluid Mechanics Conference, AFMC, Gold Coast, Australia, 39-53.

Lennon, J. M., and Hill, D. F. (2006). "Particle image velocity measurements of undular and hydraulic jumps.” Journal of Hydraulic Engineering, 132(12), 1283-1294.

Leutheusser, H. J., and Kartha, V. C. (1972). "Effects of inflow condition on hydraulic jump." Journal of the Hydraulics Division, 98(HY8), 1367-1384.

Lin, C., Hsieh, S. C., Kuo, K. J., and Chang, K. A. (2008) "Periodic oscillation caused by a flow over a vertical drop pool.” Journal of Hydraulic Engineering,134(7). 948-960.

Liu, M., Rajaratnam, N., and Zhu, D. (2004). "Turbulence structure of hydraulic jumps of low Froude numbers." Journal of Hydraulic Engineering, 130(6), 511-520.

Long, D., Steffler, P., and Rajaratnam, N. (1990). "LDA study of flow structure in submerged hydraulic jump." Journal of Hydraulic Research, 28(4):437-460.

Mehrotra, S. C. (1976). "Length of hydraulic jump." Journal of the Hydraulics Division, 102(HY7), 1027-1033.

Murzyn, F., Mouaze, D., and Chaplin, J.R., (2005). "Optical fibre probe measurements of bubbly flow in hydraulic jumps." International Journal of Multiphase Flow, 31, 141-154.

Rajaratnam, N. (1965). "The hydraulic jump as a wall jet." Journal of the Hydraulics Division, 91(HY5), 107-132.

Rajaratnam, N., and Subramanya, K. (1968). "Profile of the hydraulic jump." Journal of Hydraulics Division, 94(HY3), 663-673.

Rajaratnam, N. (1976). Turbulent jets. Elsevier Scientific Publishing Company, Amsterdam.

Ryu, Y., Chang, K. A., and Lim, H. J. (2005). "Use of bubble image velocimetry for measurement of plunging wave impinging on structure and associated greenwater." Measurement Science and Technology, 16, 1945-1953. 